\title{
The Effect of Eicosapentanoic Acid Administration on Inflammatory Markers and Clinical Outcome of Patients with Major Depression: A Pilot Study
}

\author{
El Baz A.H. ${ }^{1, *}$, El Wakeel L.M. ${ }^{2}$, El-Shafie T. M $^{3}$, Zaki M.A \\ ${ }^{1}$ Department of Clinical Pharmacy, Ahram Canadian University \\ ${ }^{2}$ Department of Clinical Pharmacy, Faculty of Pharmacy, Ain Shams University \\ ${ }^{3}$ Department of Psychiatry, Faculty of Medicine, Al Azhar University \\ *Corresponding author: elbaz_alaa@yahoo.com
}

Received November 01, 2019; Revised December 03, 2019; Accepted December 10, 2019

\begin{abstract}
Background. Major depressive disorder (MDD) affects approximately 10\% of the world population and leads to significant disability. The current study aimed to evaluate the impact of Omega3 polyunsaturated fatty acids (PUFAs) administration on the clinical outcome and inflammatory markers of patients with depression. Patients and Methods. A prospective, randomized controlled study conducted at the outpatient clinics of Alzahraa University Hospital, Cairo, Egypt on patients diagnosed with depression according to a strict inclusion and exclusion criteria. Forty-two patients were randomly assigned to either; Group1; (intervention n=21); received the prescribed antidepressant + omega3 (2100mg) for 8 weeks, or Group 2; (control, $n=21$ ); received the prescribed antidepressant only for 8 weeks. Baseline evaluation and 8-week assessment included; patient demographic data collection, history taking and clinical assessment of Diagnostic statistical manual for mental disorders, fifth edition (DSM-5) criteria \& Hamilton rating scale for depression (HAM-D score). Laboratory assessment included tumor necrosis factor- $\alpha$ (TNF- $\alpha$ ) levels. Patients were followed up regularly every week for 8 weeks for the occurrence of side effects due to antidepressants/ Omega 3 and compliance with medications. Results. The 2 groups were comparable at baseline. The test group showed a significant improvement in the HAM-D score $\&$ a reduction in TNF- $\alpha$ levels from baseline values and versus the control. There was no significant difference in the reported side effects between the 2 groups. Conclusion. Omega -3 PUFAs administration at a dose of $2100 \mathrm{mg}$ for 8 weeks, improved depression symptoms and reduced inflammatory markers \& was well tolerated.
\end{abstract}

Keywords: Omega-3 PUFAs, depression, TNF- $\alpha$, EPA/DHA

Cite This Article: El Baz A.H., El Wakeel L.M., El-Shafie T. M, and Zaki M.A, "The Effect of Eicosapentanoic Acid Administration on Inflammatory Markers and Clinical Outcome of Patients with Major Depression: A Pilot Study.” Journal of Food and Nutrition Research, vol. 7, no. 12 (2019): 815-820. doi: 10.12691/jfnr-7-12-1.

\section{Introduction}

Major depressive disorder (MDD) nearly affects a tenth of the global population. According to the Diagnostic and Statistical Manual of Mental Disorder, Fifth Edition (DSM-5, 2013), MDD is characterized by the loss of interest in pleasure, low self-esteem, disturbed sleep or fatigue, appetite, and reduced ability to think or concentrate. These problems mostly become chronic and recurrent and might lead to suicide [1].

The World Health Organization (WHO) has ranked depression as the fourth leading cause of disability worldwide and projects that, by 2020, it will be the second leading cause [2]. The lifetime prevalence of depression in urban and rural Egyptian populations found to be 11.4 and $19.7 \%$ [3].
Antidepressants have side effects that can affect compliance and morbidity. So patients are increasingly using complementary and alternative medicine therapies to treat depression [4]. One such possibility is the use of omega-3 polyunsaturated fatty acids (PUFAs) for the treatment of depression. A link between omega-3 fatty acids and mood disorders has been suggested by some studies showing a lower incidence of depression among populations with a diet rich in omega-3 fatty acids [5].

Omega-3 PUFA has been proposed, for treatment of Major depressive disorder. The positive effects of omega-3 PUFA on depression may rely on their physiological abundance in the human nervous system and their involvement in neurogenesis and neuroplasticity [6]. Moreover, their anti-inflammatory capacity may neutralize the inflammatory processes that occur in depression [7].

It has been previously documented that Eicosapentanoic acid (EPA) at ratios $>60 \%$ can positively affect 
depression outcome. EPA and Docosahexanoic (DHA) are similar in structure and might be expected to compete in around a 1:1 ratio for binding sites. Thus the amount of EPA unopposed by DHA may be critical for effective PUFA supplementation in the treatment of depressive episodes [8].

Hence the aim of the current study was to evaluate the impact of Omega-3 PUFAs administration (using an EPA in a dose more than twice the concentration of DHA), on the clinical outcome and inflammatory markers of patients with depression.

\section{Patients and Methods}

The current study was a prospective, randomized controlled study conducted at the outpatient clinics of a University Hospital (Al Zahraa), in Cairo, Egypt. All patients presenting to the outpatient clinic of the Department of Psychiatry at Al Zahraa University Hospital were assessed for eligibility according to the following inclusion and exclusion criteria. Inclusion criteria included; an age range of (18-65 years); a diagnosis of depression according to the DSM-5; and a Hamilton Depression Rating Scale (HAM-D) score of greater than or equal to 17. Exclusion criteria included having any of the following: a psychotic or seizure disorder, a current drug/ alcohol abuse or dependence, a history of drug or alcohol abuse or dependence within the previous six months, an unstable medical or neurological condition that is likely to interfere with the treatment of depression, pregnancy, history of allergy to omega-3 fatty acids, or shellfish, concomitant therapy with psychotropic medications, active suicidal ideation or other safety concerns, exposed to treatment with fluoxetine or monoamine oxidase inhibitors (MAOIs) in the previous two months; on anticoagulant therapy; receiving dietary intake of omega-3 at baseline; or refusal to participate in the study

Signed informed consent was obtained from all participants and the study design was approved by the Ethical Research Committee of the Faculty of Pharmacy, Ain Shams University, registration number \#61. All procedures performed in the study which involved human participants agreed with the ethical standards of the Institutional and/or National Research Committee and with the 1964 Helsinki declaration and its later amendments or comparable ethical standards.

The current study recruited 42 patients with a 1:1 randomization; the small sample size is justified by the fact that we are just piloting the idea to test its feasibility.

Forty eligible patients were randomly assigned to either:

Group 1; (intervention, $n=21$ ); received the prescribed antidepressant + Ocean blue professional omega3 2100 (EPA $1350 \mathrm{mg}$, DHA $600 \mathrm{mg}$ and $150 \mathrm{mg}$ of other omega 3 fatty acids) for 8 weeks.

Group 2; (control, $n=21$ ); received the prescribed antidepressant only for 8 weeks.

At Baseline patients in both groups were all subjected to the following;

-Patient demographic data collection, history taking and clinical assessment including, assessment of DSM-5 criteria by psychiatric interview \& clinical pharmacist assessment of HAM-D score to assess the severity of depression.

- Laboratory assessment of TNF- $\alpha$ levels using ELISA technique (Catalog No. ET2010-1).

Patients were followed up regularly every week for 8 weeks for assessment of the following: HAM-D score, the occurrence of side effects due to antidepressants/ Omega 3 and compliance with medications.

At the end of the study (after 8 weeks), both groups were reassessed for the following; TNF- $\alpha$ levels and depression outcome using the HAM-D score.

- HAM-D score was interpreted using the following scores; 0-7 indicated no depression; 8- 16, mild depression; $17-23$, moderate depression, and $>23$, indicating severe depression [9].

Response to treatment was defined as at least a $50 \%$ reduction in the HAM-D score, while "Remission" is a return to a normal state or a HAM-D of 7 or less.

The primary endpoint was comparing the differences in TNF- $\alpha$ levels from baseline to 8 weeks between both groups.

The secondary endpoint was comparing the differences in HAM-D score from baseline to 8 weeks between groups.

\section{Statistical Analysis}

Data management and analysis were performed using the Statistical Package for Social Sciences (SPSS) v. 23. Numerical data were described using means and standard deviations or medians and ranges, as appropriate. Categorical data were summarized as numbers and percentages. Numerical data were explored for normality using the Kolmogorov-Smirnov test and Shapiro-Wilk test. Exploration of data revealed that some of the collected values were not normally distributed. Comparisons between both groups were done by Mann-Whitney test for nonparametric data. The change between baseline and end of the study was tested using the Wilcoxon Rank Signed for nonparametric data. To measure the strength of association between the measurements, Spearman's correlation coefficients were calculated. For comparisons of parametric data, repeated measure ANOVA test was applied. All p-values were two-sided. P-values $<0.05$ were considered significant.

\section{Results}

From February 2015 till August 2016, out of a total of 75 depression patients screened, only 42 fulfilled the inclusion criteria \& were included in the study. Figure 1 shows the study flowchart.

Baseline laboratory evaluation and patients' demographics were comparable between the 2 groups, Table 1.

After 8 weeks, the intervention group showed a significant decline in the overall HAM-D score and the percentage change over time versus the control, Figure 2 and Table 1 . Serum TNF- $\alpha$ was significantly reduced in the intervention group compared to its baseline levels and compared to the control group. The percentage change over time in the TNF- $\alpha$ level showed a highly significant 
decline in the intervention group versus the control group (Figure 3 and Table 1).

Using Spearman Rho correlation, there was a highly significant positive correlation between the HAM-D score with the TNF- $\alpha$ levels, Figure 4.

Over the study period, there was no significant difference between the 2 groups in terms of the reported side effects except for the intervention group which showed fishy- aftertaste in 8 patients (38.1\%), itching in 4 patients (19\%) and GIT upset in 4 patients (19\%). These side effects were mild and self-limited and did not require drug discontinuation.

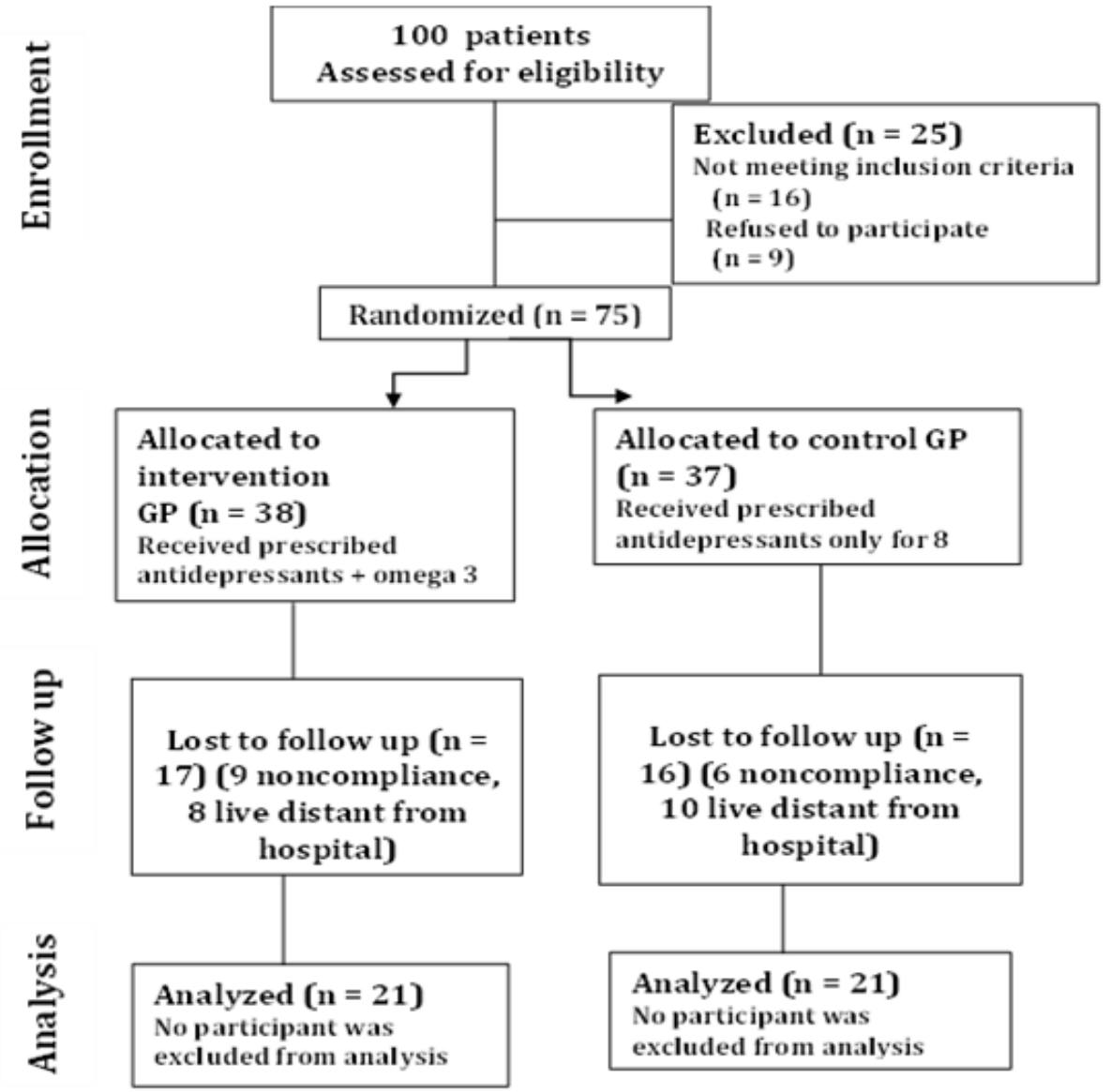

Figure 1. Study flow chart

Table 1. Comparison between control and intervention groups in demographics, laboratory and clinical parameters

\begin{tabular}{|c|c|c|c|c|c|}
\hline & \multicolumn{2}{|c|}{ Group } & \multicolumn{3}{|c|}{ P-values } \\
\hline & Control; n (21) & Intervention; n (21) & P1 & P2 & P3 \\
\hline \multicolumn{6}{|l|}{ Baseline Evaluation } \\
\hline Age (yrs); mean \pm S.D & $36.8 \pm 12.2$ & $40.8 \pm 13$ & 0.311 & & \\
\hline $\begin{array}{l}\text { Sex; male (M); n (\%) } \\
\text { Female (F); n (\%) }\end{array}$ & $\begin{array}{l}7(33.3 \%) \\
14(66.7 \%) \\
\end{array}$ & $\begin{array}{c}5(23.8 \%) \\
16(76.2 \%) \\
\end{array}$ & 0.495 & & \\
\hline \multicolumn{6}{|l|}{ Over time- Evaluation } \\
\hline \multicolumn{6}{|c|}{ HAM-D score: median (range) } \\
\hline Pre & $22(18-32)$ & 23 (18- 29) & $\$ 0.583$ & & \\
\hline Post & $10(6-15)$ & $6(2-10)$ & $\$<0.001$ & & \\
\hline $\mathrm{P} 4$ & \# 1.000 & $\#<0.001$ & & & \\
\hline$\%$ change & $53(40-70)$ & 78 (55-91) & $\$<0.001$ & & \\
\hline \multicolumn{6}{|c|}{$*$ TNF- $\alpha$ level pg/ml; mean \pm S.D } \\
\hline pre & $181.1 \pm 21.5$ & $171.6 \pm 28.4$ & 0.229 & & \\
\hline post & $111.5 \pm 11.6$ & $52.5 \pm 14.3$ & $<0.001$ & $<0.001$ & $<0.001$ \\
\hline$\%$ change & $38.1 \pm 5.1$ & $69.3 \pm 7.5$ & $<0.001$ & & \\
\hline
\end{tabular}

*, Repeated measure ANOVA

\#, Wilcoxon signed rank test

\$, Mann Whitney test

$\mathrm{P} 1=\mathrm{p}$-value for the difference between groups

$\mathrm{P} 2=\mathrm{p}$-value for the difference between pre/post

$\mathrm{P} 3=\mathrm{p}$-value for the interaction between groups and time

$\mathrm{P} 4=$ nonparametric change between pre and post values. 


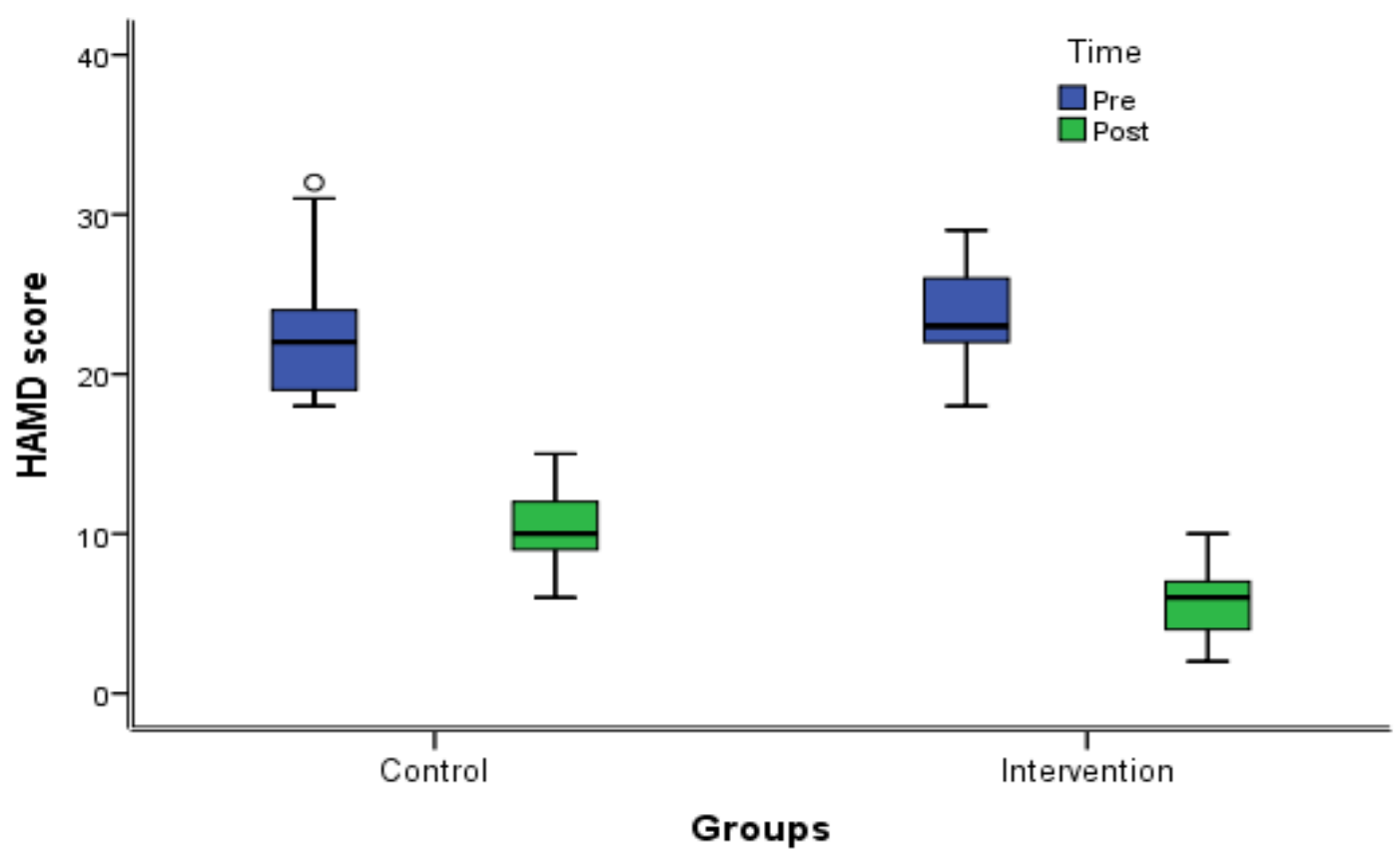

Figure 2. Comparison between pre and post HAM-D score within control and intervention Groups

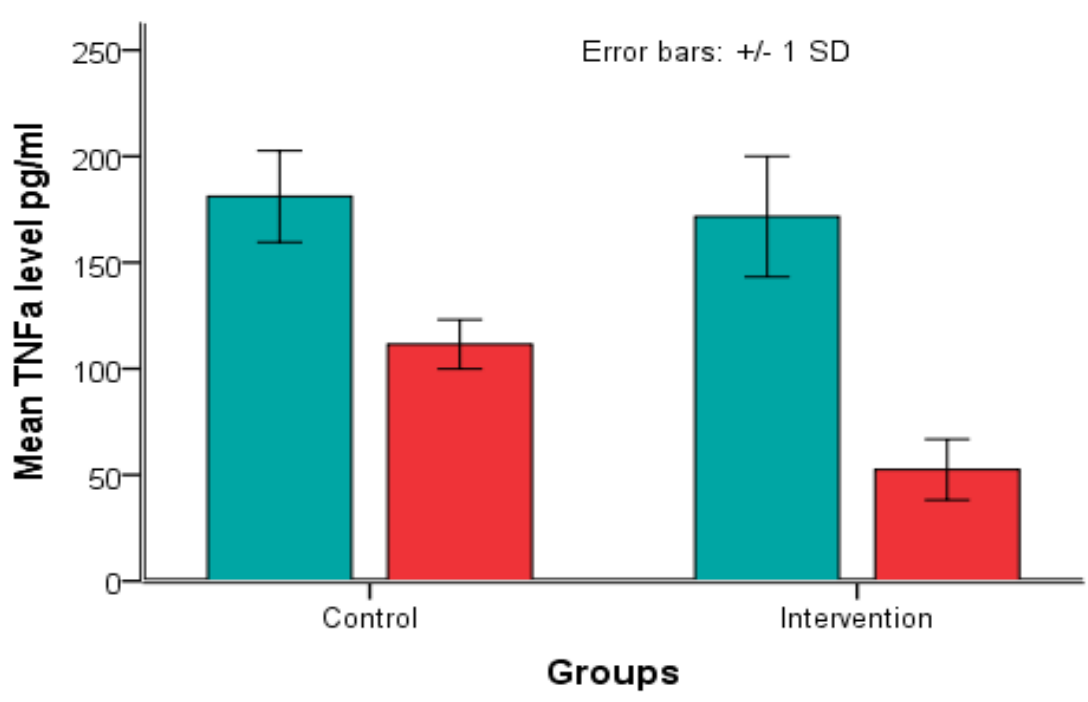

Figure 3. Comparison between pre and post TNF- $\alpha$ levels within control and intervention Groups

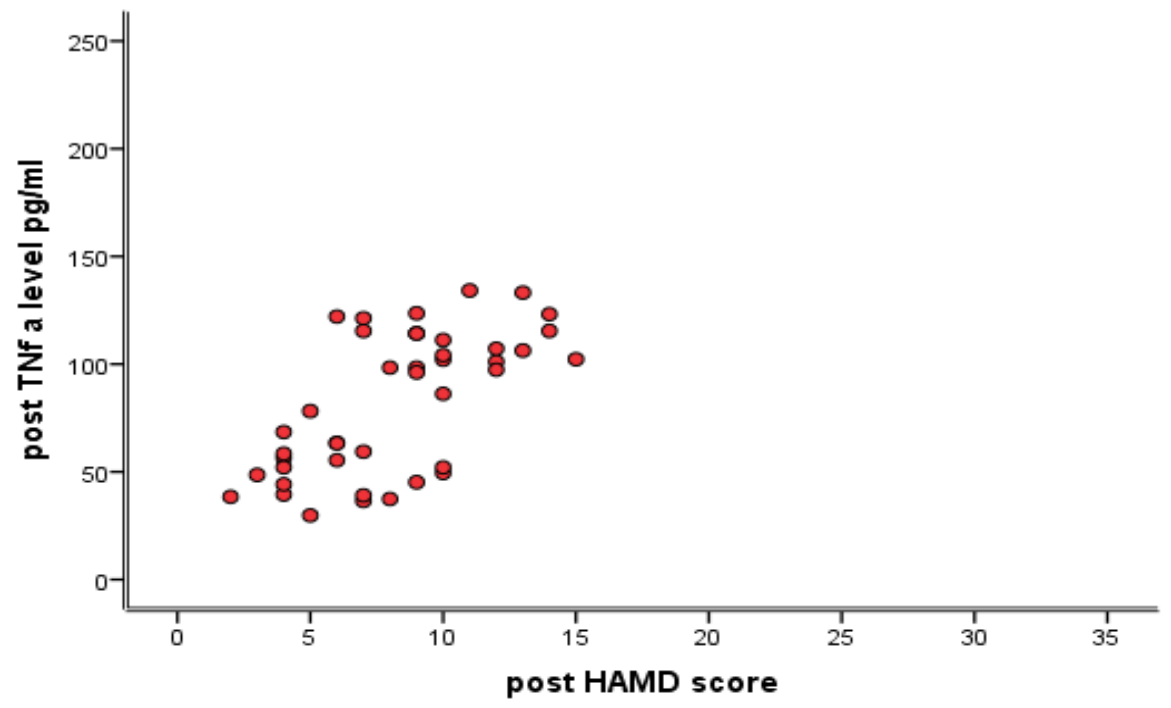

Figure 4. Correlation between the HAMD score with the TNF- $\alpha$ levels 


\section{Discussion}

Major depressive disorder (MDD) is the most prevalent Axis I disorder and one of the leading causes of disability worldwide [10].

Pharmacotherapy is the mainstay of treatment for depression, yet an adequate response to a single antidepressant medication, only occurs in about 50\%-75\% of patients. Moreover, remission rates are even less and do not usually exceed $30 \%$. Hence, the search for a naturally occurring, inexpensive, safe, and effective agent continues. The use of omega-3 polyunsaturated fatty acids (PUFAs) for treatment of depression is a potential option [5].

The results of the current study showed that the addition of omega 3 fatty acids to the standard antidepressant treatment was associated with improvement of the HAM-D score and a reduction on TNF- $\alpha$ levels.

The current study used $2100 \mathrm{mg}$ omega 3 fatty acids, containing EPA of twice the concentration of DHA in addition to the traditional selective serotonin reuptake inhibitors used for depression. The rationale for this high concentration of EPA is the previous evidence reporting that this ratio can positively affect depression outcome [8].

Several biomarkers of inflammation have been measured in MDD. One of them is TNF- $\alpha$ which has been shown to influence neurotransmitter metabolism, neuroendocrine function, and regional brain activity, all of which are relevant to depression [11]

In the current study administration of omega 3 fatty acids resulted in a significant improvement in TNF- $\alpha$ levels as compared to baseline values versus control. This was in accordance with the results of the study conducted by Glaser and colleagues on $1^{\text {st }}$ and $2^{\text {nd }}$ medical students. Their study demonstrated a significant decrease in TNF- $\alpha$ levels [12].

Similar findings were observed in a study conducted by Gharekhani and colleagues, they evaluated the effects of omega-3 fatty acids on depression and chronic inflammation in hemodialysis patients. The omega-3 supplemented group showed both a lower Beck Depression Inventory (BDI) score and a significant reduction in several inflammatory markers as TNF- $\alpha$ [13].

In contrast to these studies, another study was done by Mocking et al. They have found that supplementation of EPA did not influence inflammatory markers or depression symptoms. These results could be attributed to the presence of co-morbid disease (DM), small sample size (12 intervention and 12 control) and also low omega 3 PUFAs dose (1g/day EPA) in this study [14].

In terms of clinical outcome, in this study, baseline HAM-D score was comparable in both groups. The omega 3 fatty acids group showed a significant improvement in HAM-D score levels as compared to baseline values and to the control.

Similarly, Rapaport et al. reported significant changes in HAM-D-17 from baseline to treatment week 8 between intervention and placebo group [15]. In addition, Gertsik, et al also evaluated MDD patients receiving combination therapy of citalopram + omega 3 versus citalopram. The first combination was more effective than monotherapy in improving the HAM-D score level [5].

In contrast, Careny et al. evaluated MDD patients with CHD and reported that omega-3 fatty acids did not result in superior depression symptoms reduction (by BDI-II and
HAM-D) at 10 weeks, compared with sertraline and placebo. The discrepancy in these results with others could be attributed to the presence of CHD with depression and the smaller ratio between EPA/ EPA [16].

The current study did not report any significant side effects with Omega -3 that required intervention or therapy discontinuation. Similarly, Kiecolt-Glaser and colleagues reported non-serious adverse events with omega 3 fatty acids administration including, sore throat, nasal symptoms, stomach pain, tachycardia, and diarrhea with non-significant differences between intervention and control group [12]. In a review by Kris-Etherton et al, it was reported that the FDA has ruled that intakes of up to 3 g/d of marine omega-3 fatty acids are generally recognized safe for inclusion in the diet. Perhaps the most common side effect was a fishy aftertaste [17].

\section{Conclusion}

Omega 3 PUFAs administration resulted in improvement of major depressive symptoms indicated by a HAM-D score reduction and halting of the inflammatory process evidenced by a reduction in TNF- $\alpha$ levels. Omega 3 PUFA was well tolerated and patients who experienced side effects did not require any drug-intervention, dose reduction or dose discontinuation. Omega-3 PUFAs at a dose of 2100 (EPA $1350 \mathrm{mg}$, DHA $600 \mathrm{mg}$, and $150 \mathrm{mg}$ other omega 3 fatty acids) for 8 weeks is a well-tolerated supplement that improves depressive symptoms \& reduces inflammatory markers.

\section{Key Message}

Our conducted study provided evidence that the addition of Omega 3 PUFAs to the standard antidepressant treatment was able to improve depressive symptoms, reduce inflammation \& it was highly tolerable.

\section{Source of Support}

No pharmaceutical or industrial support.

\section{Conflict of Interest}

The authors declare that they have no conflict of interest.

\section{Disclaimers}

The use of any specific brand of neurotrophic factor was not intentional in the trial .Any available type of neurotrophic factor was used regardless of the producing company.

\section{Disclosure of Funding}

No source of funding from National Institute of Health (NIH); Welcome Trust; Howard Hughes Medical Institute (HHMI) and others. 


\section{References}

[1] Song C, Shieh CH, Wu YS, Kalueff A, Gaikwad S, Su KP. The role of omega-3 polyunsaturated fatty acids eicosapentaenoic and docosahexaenoic acids in the treatment of major depression and Alzheimer's disease: Acting separately or synergistically? Prog Lipid Res. 2016; 62: 41-54.

[2] Kessler RC, Bromet EJ. The epidemiology of depression across cultures. Annual review of public health. 2013; 34: 119-38.

[3] Beshai S, Dobson KS, Adel A, Hanna N. A cross-cultural study of the cognitive model of depression: cognitive experiences converge between Egypt and Canada. PloS one. 2016; 11(3): e0150699.

[4] Qureshi NA, Al-Bedah AM. Mood disorders and complementary and alternative medicine: a literature review. Neuropsychiatric disease and treatment. 2013;9(639):58.

[5] Gertsik L, Poland RE, Bresee C, Rapaport MH. Omega-3 fatty acid augmentation of citalopram treatment for patients with major depressive disorder. Journal of clinical psychopharmacology. 2012; 32(1): 61-4.

[6] Grosso G, Galvano F, Marventano S, Malaguarnera M, Bucolo C, Drago F, et al. Omega-3 fatty acids and depression: scientific evidence and biological mechanisms. Oxidative medicine and cellular longevity. 2014; 2014.

[7] Hennebelle M, Balasse L, Latour A, Champeil-Potokar G, Denis S, Lavialle $\mathrm{M}$, et al. Influence of omega-3 fatty acid status on the way rats adapt to chronic restraint stress. PloS one. 2012; 7(7): e42142.

[8] Sublette ME, Ellis SP, Geant AL, Mann JJ. Meta-analysis: effects of eicosapentaenoic acid in clinical trials in depression. The Journal of clinical psychiatry. 2011; 72(12): 1577.

[9] Zimmerman M, Martinez JH, Young D, Chelminski I, Dalrymple K. Severity classification on the Hamilton Depression Rating Scale. J Affect Disord. 2013; 150(2): 384-8.
[10] Ferrari AJ, Charlson FJ, Norman RE, Patten SB, Freedman G, Murray CJ, et al. Burden of depressive disorders by country, sex, age, and year: findings from the global burden of disease study 2010. PLoS medicine. 2013; 10(11): e1001547.

[11] Zunszain PA, Hepgul N, Pariante CM. Inflammation and depression. Behavioral Neurobiology of Depression and Its Treatment: Springer; 2012. p. 135-51.

[12] Kiecolt-Glaser JK, Belury MA, Andridge R, Malarkey WB, Glaser R. Omega-3 supplementation lowers inflammation and anxiety in medical students: a randomized controlled trial. Brain, behavior, and immunity. 2011; 25(8): 1725-34.

[13] Gharekhani A, Khatami MR, Dashti-Khavidaki S, Razeghi E, Noorbala AA, Hashemi-Nazari SS, et al. The effect of omega-3 fatty acids on depressive symptoms and inflammatory markers in maintenance hemodialysis patients: a randomized, placebocontrolled clinical trial. European journal of clinical pharmacology. 2014; 70(6): 655-65.

[14] Mocking RJ, Assies J, Bot M, Jansen EH, Schene AH, Pouwer F. Biological effects of add-on eicosapentaenoic acid supplementation in diabetes mellitus and co-morbid depression: a randomized controlled trial. PloS one. 2012; 7(11): e49431.

[15] Rapaport MH, Nierenberg AA, Schettler PJ, Kinkead B, Cardoos A, Walker $\mathrm{R}$, et al. Inflammation as a predictive biomarker for response to omega-3 fatty acids in major depressive disorder: a proof-of-concept study. Molecular psychiatry. 2016; 21(1): 71-9.

[16] Carney RM, Freedland KE, Rubin EH, Rich MW, Steinmeyer BC, Harris WS. Omega-3 augmentation of sertraline in treatment of depression in patients with coronary heart disease: a randomized controlled trial. Jama. 2009; 302(15): 1651-7.

[17] Kris-Etherton PM, Harris WS, Appel LJ, Nutrition C. Fish consumption, fish oil, omega-3 fatty acids, and cardiovascular disease. Arteriosclerosis, thrombosis, and vascular biology. 2003; 23(2): e20-30. 